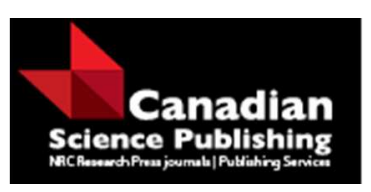

Canadian Journal of Physics

Revue canadienne de physique

\title{
Finite Gravitational Time Dilation in Black Holes Using Dynamic Newtonian Advanced Gravity (DNAg).
}

\begin{tabular}{|r|l|}
\hline Journal: & Canadian Journal of Physics \\
\hline Manuscript ID & cjp-2015-0636.R1 \\
\hline Manuscript Type: & Article \\
\hline Date Submitted by the Author: & 03-Dec-2015 \\
\hline Complete List of Authors: & $\begin{array}{l}\text { Worsley, Andrew; UCL, } \\
\text { Worsley, Joseph; Bristol University }\end{array}$ \\
\hline Keyword: & $\begin{array}{l}\text { black hole, Cygnus X-1, gravitation, Dynamic Newtonian advanced gravity, } \\
\text { time dilation }\end{array}$ \\
\hline
\end{tabular}

\section{SCHOLARONE ${ }^{\text {M }}$ \\ Manuscripts}




\title{
Finite Gravitational Time Dilation in Black Holes Using Dynamic Newtonian Advanced Gravity (DNAg)
}

\author{
Andrew Worsley ${ }^{1}$ \\ Joseph Worsley² \\ ${ }^{1}$ UCL, Gower Street, London WC1E 6BT, UK. andrew.worsley@uclmail.net \\ ${ }^{2}$ Bristol University UK. jw9525@my.bristol.ac.uk
}

Key words: gravitation, time dilation, black holes, Cygnus X-1. 


\begin{abstract}
In this paper we use a dynamic form of modified Newtonian gravity to reformulate the equations for gravitational time dilation. Here we introduce the generic equations for gravitational time dilation. It is shown that these equations agree exactly with gravitational time dilation in satellite navigation systems. The equations are also in agreement with a reanalysis of observations of gravitational red shifts in black hole accretion discs. Using these equations, we translate the time dilation into a finite value at the black hole event horizon. Thus this reformulation resolves the difficulties of the existence of black hole singularities. Importantly these dynamic gravitational equations provide testable predictions in the vicinity of black holes.
\end{abstract}




\section{Introduction:}

The current description of gravity in modern classical physics has been successful at predicting gravitational observations in low and medium gravitational fields. However, there remain some outstanding issues particularly with regards galactic rotation curves, and the presence of dark matter. We have previously shown that using a dynamic form of Newtonian advanced gravity (DNAg), it is possible to explain both the galactic rotation curves [1, 2], and the presence of dark matter [2, 3].

It is however, essential to have an understanding of time dilation and black hole gravitational physics and in particular the effects of gravitational time dilation in the vicinity of black holes. The generic equations for time dilation for this model have not previously been published. Here we present original equations for gravitational time dilation.

The current interpretation of the gravitational equations predicts that the effects of time dilation, leads to infinite time dilation at the event horizon and the formation of singularities $[4,5]$.

Classical model of gravitational time dilation:

$$
1+z=1 / \sqrt{1-\frac{2 G M}{R c^{2}}}
$$

where $M$ is the gravitational mass, $c$ is the speed of light and $G$ the gravitational constant, $R$ is the radius for space.

However, it is possible that an adaptation to the gravitational equations can be made, which can account for time dilation in the presence of black holes. To do this it is first possible to parameterize the gravitational equations for space and time separately. 
For space, the perihelion advance, in Straumann [6] is given by:

$$
\Delta \varphi \approx \tan \Delta \varphi=\frac{6 \pi m^{2}}{L^{2}}
$$

where $m=G M / c^{2}$ and $L^{2} / m=a\left(1-e^{2}\right), a$ is the semi-major axis and $e$ is the eccentricity.

This equation has been previously parameterised to account for space, and matter radius reductions in the form of dynamic gravity [3].

Space Radius $\left(R^{\prime}\right)$ and Matter Radius $\left(r^{\prime}\right)$ Reduction

$$
\begin{aligned}
& R^{\prime}=\frac{R}{\left(1+3 G M / R c^{2}\right)}, \\
& r^{\prime}=\frac{r}{\left(1+G M / 3 r c^{2}\right)},
\end{aligned}
$$

where $M$ is the gravitational mass, $c$ is the speed of light and $G$ the gravitational constant, $R$ is the radius for space.

It has previously been shown that $R^{\prime}$ gives answers that are technically in exact agreement in the advance in the perihelia of Mercury, and the other planets in the solar system. We have also used data for the binary pulsar PSR B 1534+12 from Straumann to verify the results [6]. Using Eqs. $(3,4)$ we compared this with the DD model (for Damour and Deruelle) [7, 8], and other classical models [9]. The models in general, gave results that were largely indistinguishable from that of observation for binary pulsars $[6,10,11]$.

The subject of time dilation has not previously been addressed, using the model introduced here. Extrapolating from the above principles, in this paper we further develop an adaptation for gravitational time dilation. Here, we introduce equations which are shown to account very accurately for the gravitational time 
dilation seen in GPS data (see Results 3.1), and in the extreme gravitation of black holes (see Results 3.2). Equally, these equations also resolve the problems associated with infinite time dilation and the formation of black hole singularities. Importantly these gravitational equations offer readily testable predictions for gravity, particularly in the vicinity of black holes. 


\section{Methods:}

All mathematical calculations follow strict standard algebraic and standard mathematical rules. The principal physics proofs are based upon standard physical formulae. The worked example is technically in exact agreement with observation of time dilation as seen in satellite navigation systems. This is also in agreement with data from black holes (see Results $3.1 \&$ 3.2). The paper also proposes observational experimental methods for further verification, based upon an analysis of the data from black holes, as listed in the conclusions. 


\section{1 Results: Gravitational time dilation in satellite navigation systems.}

Here we explore the technique of translating the equations for gravity from describing curved space-time, to describing a separate parameter for the equations of gravitational time dilation.

Straumann's calculations showed that an algebraic equation [Eq. (2)], was a very accurate representation of gravitational experiment, when applied to solar system and even using binary pulsar data [6]. Thus by using these principles it has also been possible to reformulate the equations for gravitational time dilation.

Here we introduce the generic equations for gravitational time dilation.

$$
\begin{aligned}
& \text { For Gravitational Time Dilation }\left(t^{\prime}\right) \\
& t^{\prime}=\quad t_{0}\left(1+G M / R c^{2}\right)
\end{aligned}
$$

where $t_{0}$ is the time measured in a zero gravitational field, $M$ is the major mass, $c$ is the speed of light and $G$ the gravitational constant, and $R$ is the radius.

In Eq. (5), the time dilation applies to objects in the presence of mass systems with one major mass $\mathrm{M}$. These observations can be very accurately applied to low mass systems relevant to GPS systems in circular orbit around Earth, as shown by a worked example below.

Calculation of Gravitational Time Dilation relative to GPS satellites (worked example).

$$
\Delta_{t}=\frac{G M}{R c^{2}}
$$

For Earth bound observers:

$$
\Delta_{t}=\frac{G M_{E}}{R_{E} c^{2}}=6.96107 \times 10^{-10}
$$


The total radius $R$ is given as the altitude of GPS $=20,180 \mathrm{~km}$, plus the averaged radius of the Earth $R_{E}=6,371 \mathrm{~km}$. Thus for the GPS satellite $R_{\text {sat }}=26,551 \mathrm{~km}$ :

$$
\Delta_{t}=\frac{G M_{E}}{R_{\text {sat }} c^{2}}=1.67033 \times 10^{-10}
$$

The time difference for the satellite is:

$6.96107 \times 10^{-10}-1.67033 \times 10^{-10}=5.2907 \times 10^{-10}$

Multiplied by the number of seconds in a day 86,400

$$
\Delta_{t}=45.7 \mu \mathrm{sec} / \text { day }
$$

Observed gravitational time dilation relative to GPS, $\Delta_{\mathrm{t}} \approx 45 \mu \mathrm{sec} /$ day.

where $\Delta_{t}$ is the change in time, $G$ is the gravitational constant, $M_{E}$ is the mass of the Earth, $R_{E}$ is the radius of Earth, $R_{\text {sat }}$ is the total orbital satellite Radius, $c$ the speed of light.

For elliptical orbits the following generic equation for time dilation applies

$$
\begin{aligned}
& \text { averaged for elliptical orbits } \\
& t^{\prime}=\quad t_{0}\left[1+G M / a\left(1-e^{2}\right) c^{2}\right],
\end{aligned}
$$

where $t_{0}$ is the time measured in a zero gravitational field, $t^{\prime}$ is the time dilation in a gravitational field, $M$ is the major mass, $c$ is the speed of light and $G$ the gravitational constant, and $a\left(1-e^{2}\right)$ where $a$ is the semi major axis and $e$ is the eccentricity. 


\section{2. Results: Gravitational time dilation in black hole systems.}

As will be shown here, in very high density objects such as black holes these equations also give very accurate results for time dilation. In doing so, they also avoid the formation of black hole singularities. Here, we develop these equations for gravitational time dilation in the vicinity of a black hole. This again gives answers that are technically in exact agreement with observations.

The principal difference in dynamic gravitation, enters the equations in the very high mass density scale. In objects such as black holes, conventionally these form an infinitely dense singularity, and classically there is infinite time dilation at the event horizon $[4,5]$. The advantage of this new approach is that we can resolve the concept of singularities and infinite time dilation. It is possible to use the dynamic advanced equations, and importantly these agree with current observation of black holes.

For the Event Horizon $\left(t_{R s}^{\prime}\right)$

$$
R_{S}=\frac{2 G M}{c^{2}},
$$

and as

$t^{\prime}=t_{0}\left(1+G M / R_{S} c^{2}\right)$,

Finite time dilation at the Schwarzschild radius of the black hole $\left(t_{R s}\right)$.

$$
t^{\prime}{ }_{R s}=t_{0} 1.5
$$

where $t_{0}$ is the time measured in a zero gravitational field, $M$ is the mass of the black hole, $c$ is the speed of light and $G$ the gravitational constant, $R_{S}$ is the distance, (taken as the Schwarzschild radius). 
If we do the calculation using DNAg, by inserting the Schwarzschild radius in to the term $R$, the actual time dilation at the event horizon in a Schwarzschild black hole is: $t_{R s}^{\prime}=t_{0} 1.5$. This would be the normal passage of time, slowed by a factor of 1.5, [Eq.(9)].

Utilising the dynamic advanced equations, it becomes possible to estimate the time dilation exerted at the Schwarzschild radius. The addition of an extra term for time elapsed in DNAg, can then also be translated to make the correction for time dilation in the vicinity of a black hole (Table 1).

\begin{tabular}{lll}
\hline $\begin{array}{l}\text { Schwarzschild } \\
\text { Radius }\left(R_{S}\right)\end{array}$ & \multicolumn{1}{c}{ GNAg } & $\begin{array}{r}\text { Gravitational Time Dilation }\left(t^{\prime}\right) \\
\text { Classical Gravity }\end{array}$ \\
\hline $1.0 R_{S}:$ & $t^{\prime}=t_{0} 1.50$ & $t^{\prime}=t_{0} \infty$ \\
$1.05 R_{S}:$ & $t^{\prime}=t_{0} 1.47$ & $t^{\prime}=t_{0} 4.58$ \\
$1.1 R_{S}:$ & $t^{\prime}=t_{0} 1.45$ & $t^{\prime}=t_{0} 2.45$ \\
$1.2 R_{S}:$ & $t^{\prime}=t_{0} 1.42$ & $t^{\prime}=t_{0} 1.73$ \\
$1.5 R_{S}:$ & $t^{\prime}=t_{0} 1.33$ & $t^{\prime}=t_{0} 1.41$ \\
$2.0 R_{S}:$ & $t^{\prime}=t_{0} 1.25$ & $t^{\prime}=t_{0} 1.29$ \\
& $t^{\prime}=t_{0} 1.20$ & $t^{\prime}=t_{0} 1.12$ \\
$10 R_{S}:$ & $t^{\prime}=t_{0} 1.10$ & $t^{\prime}=t_{0} 1.05$ \\
$5.0 R_{S}:$ & $t^{\prime}=t_{0} 1.05$ &
\end{tabular}

Table 1: Gravitational time dilation $\left(t^{\prime}\right)$ in the vicinity of black hole systems, measured in multiples of the Schwarzschild radii $\left(R_{S}\right)$ from the major black hole. Where, $t_{0}$ is the time measured in a zero gravitational field. Answers are given to 2 decimal places. 
In low and medium gravitational fields the results of current classical models and dynamic gravity presented here are technically exactly the same (see Results 3.1). Notably, in both gravitational models time dilation increase significantly as the radius of orbit approaches 10 Schwarzschild radii or less. At these distances the results still remain almost the same. Current classical gravity gives a time dilation factor of 1.05 , compared to 1.054 in dynamic advanced gravity at $10 R_{S}$. However, in classical gravity time dilation increases exponentially as we approach the event horizon, and time stops at the event horizon. With advanced gravity in the case where the event horizon has been reached at $1 R_{S}$, time dilation due to gravity is equivalent to a factor of 1.5 [Eq. (9)]. Importantly, the presumed singularities that appeared in current gravity do not appear in these dynamic advanced equations.

These observations are strongly corroborated by a re-analysis of recent observations from black holes. These observations include emissions from the radius of innermost stable circular orbit (RISCO) [12], and broadening of the iron $\mathrm{K}$ alpha line [13]. From the observations of Cygnus $X-1$, the results give an inner radius $R_{i n}=$ $1.12 \mathrm{R}_{\mathrm{S}}$, and X-ray fluorescence was shown to be predominantly confined to $10 \mathrm{Rs}$ [12]. Observations of Cygnus X-1 spectra and the iron $\mathrm{K}$ alpha line, gives a red shift $\chi^{2}{ }_{\text {red }}=1.08-1.10$ [13]. Current classical gravity [Eq. (1)], predicts an increase by a factor of 3.055 in the gravitational red shift at the radius of $1.12 \mathrm{R}_{\mathrm{s}}$, with a red shift $\chi^{2}$ red $=1.19$. Thus, the results from classical gravity predict almost twice the observed values. Here we predict gravitation red shift by a factor of 1.446 , at $1.12 \mathrm{R}_{\mathrm{S}}$ with a red shift, $\chi^{2}$ red $=1.013$, in keeping with recent observations [13].

Importantly these equations allow further accurate tests of gravitation, particularly in the vicinity of black holes. 


\section{3: Conclusions and Discussion}

The principal findings in this paper are, that using a dynamic reformulation of gravity, we can formulate the equations in a way which technically agrees exactly with gravitational experiment. This is particularly applies to the timing of GPS systems. Here we also present the generic formulae for time dilation, which allow these equations to be used in instances including that of black hole gravitational systems. Here we further show, in extreme cases such as at the Schwarzschild radius of black holes, these dynamic advanced equations can be applied to gravitational time dilation. Equation (9), indicates that time dilation at the Schwarzschild radius, where time is slowed, is not infinitely slowed but slows by a factor of 1.5 . Thus, using this Eq. (9), in the DNAg model the time dilation is not infinite, and does not result in a black hole singularity.

In previous publications we have already calculated the advance in the perihelion of Mercury and other planets and astronomical objects in the solar system, to a very high degree of accuracy [3]. A reformulation of the equations shows that advanced gravitational equations are also very accurate for binary pulsars, using recent data for PSR B 1534+12 from Straumann [6, 7]. Here, it has also been shown that theses equations are accurate for observations of Cygnus X-1 [12-14]. These results suggest a straight line correlation between dynamic advanced gravitation, for low and medium mass and high density gravitational bodies.

These dynamic advanced equations, can also help explain the apparent missing mass of the galaxy, due to the presence of (primordial) black holes in the galactic halo [1, 2], and to the indirect effects that an increased force of gravity has on the galactic rotation curve. Dynamic advanced gravity can explain the presence of 
dark matter at the galactic level because of an increase in gravity that is predicted, but goes beyond this in the prediction of observations from the bullet cluster $[2,3]$.

In this paper, it has been demonstrated that dynamic advanced gravity can also explain the physical phenomena of time dilation, and technically agrees accurately with gravitational experiment. It explains the recent findings of increased gamma rays emanating from the supermassive black hole at the centre of the galaxy [15]. By resolving infinite time dilation it also resolves the difficulties that arise from the formation of infinite density singularities. Overall, Dynamic Newtonian Advanced gravity (DNAg), de facto technically gives exactly the same answers as gravitational experiment, but it is also offers a solution to a number of contemporary gravitational anomalies.

Importantly it offers a number of verifiable predictions for gravity, particularly in the vicinity of black holes. This could be achieved in accordance with the results of the generic equations for black hole as given in Table 1. Gravitational time dilation could either be measured the using the gravitational red shift of a strong EM source in the vicinity of a black hole. Equally well a probe issuing a timed signal could be used. Significant differences between DNAg and classical gravity would emerge at about 10 Schwarzschild radii. 


\section{References.}

1]. Hawkins M. R. S. The case for primordial black holes as dark matter. Mon. Not Roy. Astron. Soc. 415: 2744 (2011).

2]. Worsley A. Advances in Black Hole Physics and Dark Matter Modelling of the Galactic Halo. Appl. Phys. Res. 4: 128-137

3]. Worsley A. The formulation of dynamic Newtonian advanced gravity, DNAg. Can. J. of Phys. 92: 1485-1488 (2014)

4]. Misner C.W., Thorne K.S. and Wheeler J.A. Gravitation. W.H. Freeman \& Co., (1973).

5]. Peacock J.A. Cosmological Physics. Cambridge University Press (1999).

6]. Straumann N. General Relativity with Applications to Astrophysics. SpringerVerlag (2004).

7]. Damour T., Deruelle N. Ann Inst H Poincaire (Physique Theoretique) 43: 107 (1985).

8]. Damour T., Deruelle N. Ann Inst H Poincaire (Physique Theoretique) 44: 263 (1986).

9]. Stairs L. H., Thorsett S.E., Taylor J.H. Wolszczan A. Studies of the Relativistic Binary Pulsar PSR B1534+12. I. Timing Analysis. Astrophys. J. 581: 501 (2002).

10]. Worsley A. String quintessence and the formulation of advanced quantum gravity. Phys. Essays. 22: 364-377 (2009).

11]. Ohanian H.C, Ruffini R. Gravitation and Spacetime. W. W Norton \& Co., 2nd Edition (1994).

12]. Gou L. et al. The extreme spin of the black hole in Cygnus X-1. The Atrophys J. 742: 85 (2011).

13]. Duro R. et al. The broad iron $\mathrm{K}$ alpha line of Cygnus $\mathrm{X}-1$ as seen by XMM-Newton in the EPIC-pn modified timing mode. Astron. \& Astrophys. 533: L3 (2011)

14]. Worsley A. Corroboration of Dynamic Black Hole Gravitational Physics from observations of Cygnus X-1. Applied Physics Research. 4: 159-165 (2012).

15]. Berlin A., Hooper D., McDermott S., Simplified dark matter models for Galactic centre gamma ray excess. Phys. Rev. D. 89: 42001 (2014). 